\title{
Nutritional Steps to Maintain Health and Reduce Cancer Risk
}

\author{
Alfred Ordman \\ Beloit College, Beloit, USA \\ Email: ordman@beloit.edu
}

How to cite this paper: Ordman, A. (2019) Nutritional Steps to Maintain Health and Reduce Cancer Risk. Journal of Cancer Therapy, 10, 829-834.

https://doi.org/10.4236/jct.2019.1010070

Received: September 23, 2019

Accepted: October 15, 2019

Published: October 18, 2019

Copyright $\odot 2019$ by author(s) and Scientific Research Publishing Inc. This work is licensed under the Creative Commons Attribution International License (CC BY 4.0).

http://creativecommons.org/licenses/by/4.0/

\begin{abstract}
At the 2017 Linus Pauling meeting, it was announced that based on metabolomic analysis, vitamin $\mathrm{K}$ at levels far above the daily value activates a variety of enzymes that are beneficial. The enzymes have been shown to remove calcium where it harms our health, deposit it where it benefits bone, may reduce hip fractures, and decrease calcification of the circulatory system, reducing by $50 \%$ the risk of heart disease and high blood pressure. Linus Pauling demonstrated that vitamin $\mathrm{C}$ can kill cancer cells. One form of vitamin $\mathrm{K}$ regenerates vitamin $\mathrm{C}$ so that it is even more effective at killing cancer cells. I previously reported a trial that determined an oral dosage of vitamin $\mathrm{C}$ that produces a concentration in the bladder able to kill recurring non-invasive muscle carcinoma cells. Nature in 1999 published an article on foods that reduce the risk of cancer. Since then there is additional evidence that nutrients can extend the human healthspan. Other nutrients are effective at reducing the risk of cancer, such as the active ingredient in cruciferous vegetables. These nutrients that reduce the risk of cancer and improve health are GRAS. They are safe and likely to substantially reduce the risk of cancer, heart disease, hip fractures, and other undesirable conditions. While research on treating cancer dominates media attention, pharmaceutical development, and Federal funding, it is likely that people can prevent cancer by consuming nutrients that have been shown to kill human cancer cells.
\end{abstract}

\section{Keywords}

Vitamin K, Vitamin C, Healthspan, Cancer Prevention, Calcium, Bladder Cancer

\section{Introduction}

In 2018, nearly 2 million new cases of cancer were diagnosed in the United States and 609,640 people died from the disease [1]. Though the cancer death 
rate fell by $26 \%$ from 1991 to 2015 , the cost of treatment has increased tremendously. From 2009 to 2014, drugs were priced at more than $\$ 100,000$ per patient for one year of treatment. In 2018, launch prices have risen to more than $\$ 400,000$ a year [2].

Statements from the National Cancer Institute (NCI) discourage nutritional steps to reduce the risk for cancer: "With few exceptions, studies of human populations have not yet shown definitively that any dietary component causes or protects against cancer. Sometimes, the results of epidemiologic studies that compare the diets of people with and without cancer have indicated that people with and without cancer differ in their intake of a particular dietary component.

However, these results show only that the dietary component is associated with a change in cancer risk, not that the dietary component is responsible for, or causes, the change in risk. For example, study participants with and without cancer could differ in other ways besides their diet, and it is possible that some other differences account for the difference in cancer" [3].

This NCI report lists a variety of substances discussed in this paper, including antioxidants, tea, vitamin $\mathrm{D}$, and cruciferous vegetables. It states that while they show reduced cancer risk in animals and epidemiologically in people, no clinical trials have proven they are effective. However, as these nutrients are safe and may prevent cancer, why not encourage their consumption? This article is to inform everyone to spread the word on nutrients that preserve health.

Unfortunately, there is little financial incentive for finding a way to prevent cancer: "Despite more than 2.4 million papers published on cancer research to date, conventional medicine has largely failed to identify safe, low-cost, effective methods of cancer intervention and prevention. The great majority of cancer research is focused on curing late stage cancers that have already spread throughout the body by the time they are detected. The reasons for this heavily skewed focus are manifold. First, societies, in general, are reactive rather than proactive. Second, the final stages of treatment research (and regulatory approval) can be simpler to perform than prevention research (requiring just hundreds of patients versus tens of thousands of patients). Third, it is much more dramatic to effectively treat a patient with advanced disease than to prevent disease. Thus many patients who are effectively treated donate large sums to cancer centers; there are few thanks given for preventing cancers. Fourth, there are few financial incentives for industry to support primary prevention measures based on avoidance of risk factors. And finally, the financial incentives to develop new therapeutics are far more lucrative than those for new diagnostic tests for early detection and prevention" [4].

\section{Nutritional Steps}

Though they are not publicized, there are clear and easy steps likely to reduce your risk of cancer. In 2010, an article [5] listed specific foods that reduced the risk of cancer. These include 1) soy (for isoflavones); 2) curcumin (turmeric); 3) 
green tea (with EGCG); 4) resveratrol; 5) cruciferous veggies (with sulforaphane glucosinolate); 6) lycopene; 7) vitamin $\mathrm{D}$; and 8) selenium. Since that time, the specific chemicals in those foods, the mechanisms by which those chemicals reduce cancer risk, and additional natural substances have been added to that list.

Table 1 provides a list of nutrients that are safe and may reduce the risk of cancer. Just because they have not been absolutely proven to prevent cancer, in the Journal of Cancer Therapy it seems important to share the knowledge that they are worthwhile to consume.

Cayenne is effective at killing cancer cells, but many have unpleasant stomach side effects. Turmeric is another spice that kills cancer cells, among other likely benefits. Turmeric extract from rhizomes is mainly composed of curcumin. The research on curcumin has received considerable attention due to its pronounced anti-inflammatory, anti-oxidative, immunomodulating, anti-atherogenic, and anti-carcinogenic activities [6]. Turmeric curcumin can help promote healthy joints, keep our hearts in top shape, and maintain our cognitive functioning. Researchers are also suggesting that, when taken as a supplement, it can help to keep our immune system balanced and working correctly [5].

Extensive epidemiological evidence and animal experiments suggest that cruciferous vegetables may prevent or delay various inflammatory disorders, including cancers. Much of this chemopreventive effect has been attributed to the physiological effect of the isothiocyanates, especially sulforaphane. Sulforaphane has been proven as a potent protector against oxidative damage and carcinogens. A plethora of clinical benefits are reported in research including human clinical studies [7].

Chromium has been added to address various health conditions. Among the most active areas of chromium research are its use in supplement form to treat diabetes, lower blood lipid levels, promote weight loss, and improve body composition [8].

However, the primary nutrients expected to reduce cancer risk and recurrence are the combination of vitamin $\mathrm{C}$ and VK1. Taking $2 \mathrm{~g}$ of VC orally twice a day for two consecutive days raises the bladder concentration of $\mathrm{VC}$ to about $4 \mathrm{mM}$,

Table 1. Nutrients that are safe and likely to reduce the risk of cancer.

\begin{tabular}{cc}
\hline Nutrient & Reference \\
Cayenne & Zheng 2016 \\
Cruciferous vegetables (esp. sulforaphane glucosinolate) & Tortorella 2015 \\
Soy & Sakar 2010 \\
Tea (esp. EGCG) & Fujiki 2018 \\
Turmeric/Curcumin & Menon 2007, Gupta 2013 \\
Vitamin C & Folk 2015 \\
Vitamin D & Zhang 2019 \\
Vitamin K & Jarabek 1995 \\
\hline
\end{tabular}


where it becomes a pro-oxidant capable of killing recurring bladder cancer cells without harming normal cells [9].

VK1 taken by oral supplementation is metabolized to form VK3, which then becomes VK2 (Figure 1). Especially valuable to prevent cancer is the combination of VC and VK3. Though VK3 is not authorized for humans in supplement form, it recycles VC 20x to produce pro-oxidant activity able to kill cancer cells [10]. While oral VC alone does not reach a serum level able to kill cancer cells, the recycling by VK3 may allow it to prevent new cancer cells from surviving in humans.

All three forms of VK (K1 (phylloquinone), K2 (menaquinone), and K3 (menadione)) provide great benefit [11], including reducing the risk for heart disease, Alzheimer's, fracture risk, and much more. In this brief report, just a few examples of the evidence are provided. VK helps cardiac rhythm. VK activates the protein osteocalcin, which strengthens bone mass, but decalcifies soft tissues like arterial walls reducing blood pressure. A recent study found that patients with early-stage Alzheimer's disease consumed less VK than did cognitively intact control subjects. "For most of mankind's existence, scientists wallowed in lethal ignorance regarding the critical need for humans to supplement with enough VD. We fear the same knowledge deficit exists today regarding VK" [12].

\section{Discussion}

Too often people wait until they get the symptoms of cancer or heart disease, and require expensive treatments. It is much simpler to avoid most age-associated illnesses by avoiding environmental hazards, obtaining good nutrition and getting regular exercise. People are often disappointed because they do not feel any different when taking supplements like AREDs to prevent macular degeneration, though research demonstrates it significantly reduces the risk for macular degeneration [13]. Claims for health benefits for nutrients that are not patented are rarely possible, because obtaining authorization from the Food and Drug Administration is an expensive and lengthy process. Inexpensive nutritional supplements do not generate enough profit to finance these costs. So the public remains unaware of the value of many supplements which may benefit health. I am fortunate to have submitted a provisional patent on a tablet with the ingredients described in this paper. The funds by sales of the product can fund a clinical trial to demonstrate its effect on cancer and heart disease. Results are likely

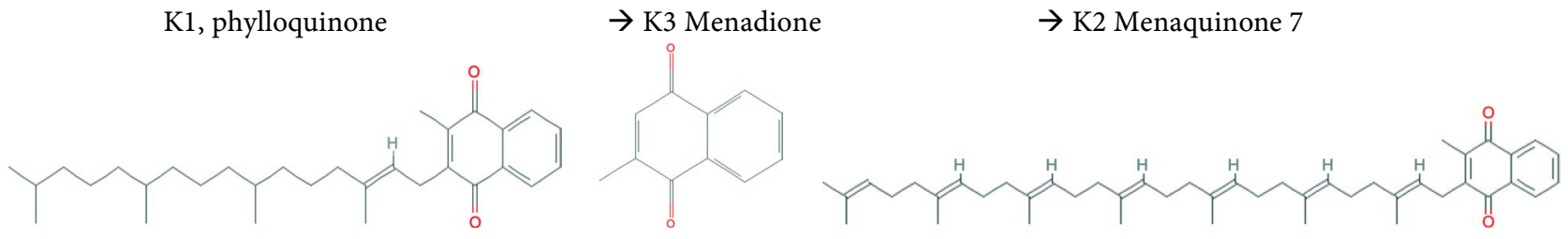

Figure 1. Three Forms and Metabolism of Vitamin K. Researchers confirmed the conversion of vitamin K1 through intermediate $\mathrm{K} 3$ to end product K2 takes place in humans [14]. Results suggest that cerebral menaquinone-4 (VK2) originates from oral phylloquinone (VK1) intake and that there are two routes of accumulation [15]. 
to support an FDA petition to make the claims of the health-promoting and disease-preventing effects of this supplement, Triumph. In this article, I present the peer-reviewed scientific evidence to support why the clinical trial is likely to show reduction in risk of many diseases associated with aging.

\section{Conclusion}

VC, VK, and other nutraceuticals mentioned in this article have all been reported in scientific research studies to have safe and proven mechanisms. Their consumption is likely to result in prolonging your healthspan. Safely, conveniently, and inexpensively reducing the risk for cancer, heart disease, and other challenges of aging is a much better choice than getting diseases and spending a fortune on pharmaceuticals and medical bills to fight them.

\section{Conflicts of Interest}

The author has filed a patent on ingredients in Triumph and will be selling Triumph once it is manufactured.

\section{References}

[1] NCI (2019a) Cancer Statistics. https://www.cancer.gov/about-cancer/understanding/statistics

[2] NCI (2019b) The Imperative of Addressing Cancer Drug Costs and Value. https://www.cancer.gov/news-events/cancer-currents-blog/2018/presidents-cancerpanel-drug-prices

[3] NCI (2015) Cancer Causes and Prevention: Diet. https://www.cancer.gov/about-cancer/causes-prevention/risk/diet

[4] Song, M., et al. (2018) Cancer Prevention: Molecular and Epidemiologic Consensus. Science, 361, 1317-1318. https://doi.org/10.1126/science.aau3830

[5] Sarkar, F.H., Li, Y., Wang, Z. and Kong, D. (2010) The Role of Nutraceuticals in the Regulation of Wnt and Hedgehog Signaling in Cancer. Cancer and Metastasis Reviews, 29, 383-394. https://doi.org/10.1007/s10555-010-9233-4

[6] Menon, V.P. and Sudheer, A.R. (2007) Antioxidant and Anti-Inflammatory Properties of Curcumin. Advances in Experimental Medicine Biology, 595, 105-125. https://doi.org/10.1007/978-0-387-46401-5_3

[7] Gupta, S.C., Patchva, S. and Aggarwal, B.B. (2013) Therapeutic Roles of Curcumin: Lessons Learned from Clinical Trials. The AAPS Journal, 15, 195-218. https://doi.org/10.1208/s12248-012-9432-8

[8] NIH (2019) Chromium: Dietary Supplement Fact Sheet. https://ods.od.nih.gov/factsheets/Chromium-HealthProfessional/

[9] Folk, E., Downs, T.M. and Ordman, A.C. (2015) Two Grams BID Is an Oral Dosage of Vitamin C to Reduce the Risk of Recurrence of Superficial Bladder Carcinoma. Journal of Cancer Therapy, 6, 169-176. https://doi.org/10.4236/jct.2015.62019

[10] Jarabek, R. and Jarabek, J. (1995) Effect of Ascorbate on the DT-Diaphorase-Mediated Redox Cycling of 2-Methyl-1,4-Naphthoquinone. Archives of Biochemistry and Biophysics, 318, 418-423. https://doi.org/10.1006/abbi.1995.1249

[11] Schwalfenberg, G.K. (2017) Vitamins K1 and K2: The Emerging Group of Vitamins Required for Human Health. Journal of Nutrition and Metabolism, 2017, Article 
ID: 6254836. https://doi.org/10.1155/2017/6254836

[12] Presse, N., Shatenstein, B., Kergoat, M.J. and Ferland, G. (2008) Low Vitamin K Intakes in Community-Dwelling Elders at an Early Stage of Alzheimer's Disease. Journal of the Academy of Nutrition and Dietetics, 108, 2095-2099. https://doi.org/10.1016/j.jada.2008.09.013

[13] Chew, E.Y., et al. (2012) The Age-Related Eye Disease Study 2 (AREDS2): Study Design and Baseline Characteristics (AREDS2 Report Number 1). Ophthalmology, 119, 2282-2289. https://doi.org/10.1016/j.ophtha.2012.05.027

[14] Thijssen, H.H., Vervoort, L.M., Schurgers, L.J. and Shearer, M.J. (2006) Menadione Is a Metabolite of Oral Vitamin K. British Journal of Nutrition, 95, 260-266. https://doi.org/10.1079/BJN20051630

[15] Okano, T., et al. (2008) Conversion of Phylloquinone (Vitamin K1) into Menaquinone-4 (Vitamin K2) in Mice: Two Possible Routes for Menaquinone-4 Accumulation in Cerebra of Mice. Journal of Biological Chemistry, 283, 11270-11279. https://doi.org/10.1074/jbc.M702971200 